\title{
Viabilidade agroeconômica do consórcio mangarito com milho verde em quatro épocas de associação ${ }^{1}$
}

\author{
Adriana Uchôa Brito ${ }^{*}$, Mário Puiatti ${ }^{2}$, Paulo Roberto Cecon ${ }^{3}$, Guilherme Alves Puiatti ${ }^{4}$
}

10.1590/0034-737X201865040009

\begin{abstract}
RESUMO
A consorciação de culturas é uma prática que permite a maximização dos recursos disponíveis, sobretudo na pequena propriedade agrícola. Objetivou-se verificar a viabilidade agroeconômica do consórcio do mangarito com milho verde. Dois experimentos foram realizados com as variedades de mangarito 'Gigante' e 'Pequeno', cada um constituído de nove tratamentos envolvendo os consórcios do mangarito com milho verde, em quatro épocas da associação após a emergência das plantas de mangarito ( $\mathrm{DAE}=0,21,42 \mathrm{e} 63$ ), assim como suas respectivas monoculturas. O delineamento experimental foi o de blocos casualizados, com cinco repetições. Avaliaram-se, além dos dados produtivos das culturas, indicadores agroeconômicos de eficiência. Os resultados foram submetidos à análise de variância e as médias de comparadas pelo teste Tukey, além de realizados contrastes (teste F) entre monocultivos solteiro e consórcios para as variáveis medidas no milho verde. Concluiu-se que é viável, agronômica e economicamente, a consorciação dos mangaritos 'Gigante' e 'Pequeno' com milho verde. Em termos econômicos, a associação do milho verde com mangarito 'Gigante' deve ser instalada, preferencialmente, aos 21 DAE das plantas do mangarito e, para o mangarito 'Pequeno', ao 0DAE.
\end{abstract}

Palavras-chave: associação de culturas; Xanthosoma riedelianum Schott; indicadores econômicos; produtividade.

\section{ABSTRACT}

\section{Agroeconomic viability of mangarito intercropped with green corn in four seasons of} association

Intercropping is a practice that allows the maximization of resources available in the agricultural property. The objective was to verify the agroeconomic viability of the mangarito intercropped with green corn. There were two experiments, conducted with the "Gigante" and "Pequeno" mangarito varieties, each one constituted of nine treatments, resulting from the intercropping of mangarito with green corn in four seasons of the association after the emergence of the mangarito plants (DAE $=0,21,42$, and 63), as well as their respective monocultures. The experimental design was a randomized block design, with five replications. In addition to crop production data, agroeconomic efficiency indicators were evaluated. Data were subjected to analysis of variance, and the means of mangarito production and of agroeconomic indicators compared by the Tukey's test, and contrasts were performed ( $\mathrm{F}$ test) between single and intercropped crops for characteristics evaluated in green corn. It is concluded that the intercropping of Gigante and Pequeno mangarito varieties with green corn is viable, both agronomically and economically. In economic terms, the association of green corn with Gigante mangarito should be installed, preferably, at $21 \mathrm{DAE}$ of the mangarito plants and, for the Pequeno variety, at 0 DAE.

Keywords: intercropping; Xanthosoma riedelianum Schott; economic indicators; yield.

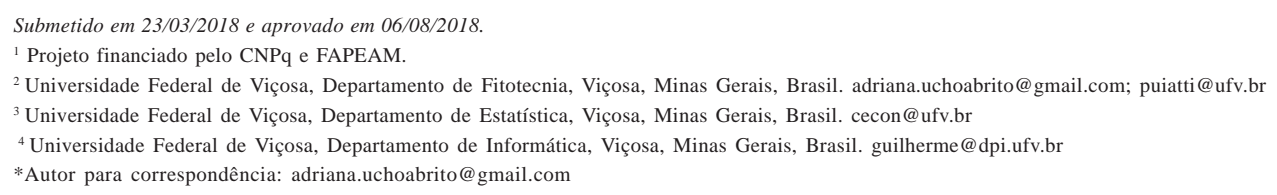




\section{INTRODUÇÃO}

A preservação da produtividade em áreas agrícolas envolve conceitos de sustentabilidade, os quais estão relacionados com o desenvolvimento econômico aliado à conservação dos recursos naturais e à qualidade de vida dos agricultores e de seus familiares. Assim, a geração e uso de práticas agrícolas conduzidas com o conhecimento detalhado dos processos ecológicos que ocorrem nas áreas produtivas, e com os conceitos mais amplos dos quais elas fazem parte, surgem como formas de aproximação da sustentabilidade (Gliessman, 2009).

O cultivo consorciado desponta como uma das práticas agrícolas alternativas para a produção de hortaliças com redução de impacto ambiental, principalmente para produtores da agricultura familiar, os quais não dispõem de grande área física para seus cultivos (Vieira et al., 2014; Brito et al., 2017). Além de proporcionar o uso mais harmônico da área agrícola, graças à diversificação das culturas, a consorciação pode proporcionar variação das fontes de renda com retorno antecipado (Heredia Zárate et al., 2006; Vieira et al., 2014; Brito et al., 2017).

A população de plantas, bem como o arranjo entre elas, tem efeito marcante sobre a produção, já que a intercompetição por água, luz e nutrientes, em plantios densos, pode contribuir para a redução da capacidade produtiva das plantas, incidindo em maior ou menor grau na produtividade das diferentes espécies (Heredia Zárate et al., 2005; 2006; 2013). Essa intercompetição pelos fatores de crescimento entre as culturas consorciadas, além de dependente das espécies consorciadas, também o é da época (estádio de desenvolvimento das culturas) em que ocorre essa associação (Vieira et al., 2014; Brito et al., 2017).

O mangarito [Xanthosoma riedelianum (Schott) Schott, ex-X. maffafa] é uma hortaliça cormosa, originária da região centro-americana, conhecida no Brasil como mangará e taioba portuguesa (Gonçalves, 2011; Brasil, 2013). É uma herbácea tuberosa com cormo e cormelos comestíveis, ereta, perene, decídua no inverno, acaule, podendo chegar a 50 centímetros de altura. Tem folhas membranáceas, glabras, de 20-25 centímetros de comprimento, com pecíolo ereto e esponjoso de 30-50 centímetros (Kinupp \& Lorenzi, 2014).

A planta apresenta caule subterrâneo principal (cormo), com brotações laterais (cormelos) e várias folhas grandes que brotam do cormo e dos cormelos. O ciclo cultural é variável, de cerca de oito a 11 meses. Durante os primeiros seis meses ocorre o desenvolvimento do cormo, dos cormelos e das folhas e, nos últimos meses, o amadurecimento de cormelos e a senescência das folhas, indicando o ponto de colheita (Costa et al., 2008; Cavalcanti, 2011; Brasil, 2013).

Embora ainda existam poucas referências quanto às necessidades nutricionais do mangarito, Costa et al. (2008), utilizando-se a técnica do elemento faltante, em Latossolo Vermelho-Amarelo, não verificaram sintomas visíveis de deficiência nutricional nas plantas do mangarito. Isso indica que a espécie pode ser pouco exigente em fertilidade do solo, apropriada para o cultivo em propriedades com limitação de recursos para aquisição de fertilizantes orgânicos ou minerais.

A densidade populacional e o arranjo das plantas são fatores relevantes para a produtividade das culturas. De modo geral, com maiores populações de plantas, a produção das culturas é reduzida pelo sombreamento das folhas baixeiras, por causa da menor quantidade de radiação solar que recebem (Rocha, 2008; Taiz \& Zeiger, 2013). Em mangarito, a população pode ser ajustada por meio do espaçamento entre as linhas e, ou, entre plantas na linha de plantio (Heredia Zárate et al., 2013; Castro et al., 2017). Todavia, melhores resultados em produtividade de mangarito foram obtidos com a maior população de plantas (Castro et al., 2017). De acordo com os autores, esse resultado pode estar relacionado com a arquitetura das folhas das plantas do mangarito, que são semieretas, sombreando-se pouco umas às outras, além de certa tolerância à restrição parcial de luz.

Características de baixa exigência nutricional, aliadas às de ciclo cultural longo e à tolerância à restrição de luz, abrem a perspectiva do potencial do cultivo do mangarito consorciado com espécies de maior porte e de menor ciclo cultural, proporcionando ao agricultor renda complementar e antecipada durante o cultivo do mangarito.

Com base no exposto, objetivou-se com este trabalho verificar as viabilidades agronômica e econômica da associação de duas variedades de mangarito com milho verde, em quatro épocas de associação das culturas.

\section{MATERIAL E MÉTODOS}

O experimento foi conduzido a campo, de 20/10/2015 a 10/07/2016, na Horta do Departamento de Fitotecnia da Universidade Federal de Viçosa (UFV). O município de Viçosa apresenta altitude de $650 \mathrm{~m}$, latitude sul de $20^{\circ} 45^{\prime}$, longitude oeste de $42^{\circ} 51^{\prime}$ e classificação climática de Köppen (1948) do tipo Cwa, caracterizado pelo clima tropical de altitude, com chuvas no verão e seca no inverno. A temperatura média durante o período experimental foi de $20,4^{\circ} \mathrm{C}$; a média das temperaturas mínimas, de $14,3^{\circ} \mathrm{C}$; e a média das temperaturas máximas, de $26,2^{\circ} \mathrm{C}$.

O solo da área de cultivo é caracterizado como Argissolo Vermelho-Amarelo câmbico, textura franco argilo-arenosa (Embrapa, 2013), cuja amostragem realizada na camada de $0-20 \mathrm{~cm}$ indicou os seguintes resultados: $\mathrm{pH}_{\text {(agua) }}=5,7 ; \mathrm{P}=42,3 \mathrm{e} \mathrm{K}=69 \mathrm{mg} \mathrm{dm}^{-3} ; \mathrm{Ca}^{2+}=1,8 ; \mathrm{Mg}^{2+}=$ $0,4 \mathrm{e} \mathrm{Al}^{3+}=0,0 \mathrm{cmol}_{c} \mathrm{dm}^{-3} ; \mathrm{B}=0,3 ; \mathrm{Fe}=75,4 ; \mathrm{Mn}=122,8 ; \mathrm{Zn}$ $=14,5 \mathrm{e} \mathrm{Cu}=4,2 \mathrm{mg} \mathrm{dm}^{-3} ;$ M.O. $=1,97 \mathrm{dag} \mathrm{kg}^{-1} ;(\mathrm{H}+\mathrm{Al})=$ 
3,$77 ; \mathrm{SB}=2,38 ; \mathrm{CTC}(\mathrm{t})=2,38 \mathrm{e} \mathrm{CTC}(\mathrm{T})=5,85 \mathrm{cmol}_{\mathrm{c}} \mathrm{dm}^{-3}$; $\mathrm{V}=41 \%$ e $\mathrm{P}-\mathrm{rem}=36,7 \mathrm{mg} \mathrm{l}^{-1}$. As análises químicas foram realizadas conforme metodologia descrita por Embrapa (2009).

Cada experimento foi constituído de nove tratamentos, constantes de quatro épocas de cultivos consorciados de mangarito (cultura principal) com milho verde, e seus respectivos cultivos solteiros (Tabela 1). Foi conduzido no delineamento de blocos casualizados, com cinco repetições.

Duas variedades de mangarito foram utilizadas, a ' $\mathrm{Gi}$ gante' e a 'Pequeno', sendo conduzidos como experimentos separados. Utilizaram-se como mudas cormelos da classe grande dos mangaritos 'Gigante' e 'Pequeno', provenientes do Banco de Germoplasma de Hortaliças da UFV, com massas média de 10,0 e de $6,0 \mathrm{~g}$, respectivamente. As sementes de milho verde utilizadas foram da variedade 'AL Bandeirante' (CATI, 2018), destinada à agricultura familiar.

O plantio do mangarito foi realizado em sulcos distanciados de $0,80 \mathrm{~m}$, abertos à profundidade de $0,10 \mathrm{~m}$, onde foram colocadas as mudas (cormelos) a cada $0,25 \mathrm{~m}$ e cobertas com solo. $\mathrm{O}$ milho foi semeado em quatro épocas pré-estabelecidas, ou seja, aos 0, 21, 42 e 63 dias após a emergência do mangarito (DAE). Considerou-se como zero dia quando, pelo menos, $90 \%$ das plantas de mangarito estavam com a parte aérea emergida do solo, o que aconteceu cerca de 30 dias após o plantio do mangarito.

Quando cultivadas solteiras ou em consórcio, as plantas de milho foram distribuídas na mesma linha de plantio do mangarito, distanciadas de $0,80 \mathrm{~m}$ entre linhas e, individualmente, a cada $0,25 \mathrm{~m}$ na linha. As sementes foram colocadas em covas de $0,05 \mathrm{~m}$ de profundidade, abertas entre as plantas de mangarito, e cobertas com solo. Foram semeadas três sementes de milho por cova, procedendo-se o desbaste 15 dias após a semeadura, deixando-se apenas uma planta a cada 0,25 m. Dessa forma, os cultivos solteiros ou em associação tiveram as mesmas populações (associação aditiva), ou seja, 50.000 plantas ha $^{-1}$ de mangarito e, ou, plantas de milho verde.

A parcela experimental foi constituída de quatro linhas, espaçadas de $0,80 \mathrm{~m}$ com $2,5 \mathrm{~m}$ de comprimento, contendo dez plantas por linha, totalizando 40 plantas de mangarito e, ou, milho, em uma área total de $8 \mathrm{~m}^{2}$ por parcela. Considerou-se como área útil, nas avalições, a área ocupada por 12 plantas de mangarito e, ou, de milho verde, nas duas fileiras centrais da parcela.

A fertilização do solo foi realizada para cada cultura, com base nas análises química e física do solo e na recomendação para o Estado de Minas Gerais (Ribeiro et al., 1999). Dessa forma, os tratamentos em consórcio receberam as adubações recomendadas para ambas as culturas, conforme Cecílio Filho et al. (2008) e Vieira et al. (2014). Em razão da ausência de recomendação de adubação para a cultura do mangarito, utilizou-se metade da dose recomendada para a cultura do taro (Colocasia esculenta L.).

O controle das plantas daninhas foi realizado mecanicamente utilizando enxada. Na ausência de chuvas e até 30 dias antes da colheita do mangarito, os cultivos receberam irrigações semanais, por aspersão, aplicando-se, em média, em cada irrigação, lâmina de água de cerca de $30 \mathrm{~mm}$ por irrigação, suficiente para atender à demanda das culturas.

A colheita das espigas foi realizada quando os grãos apresentavam-se em fase leitosa (estádio de "pamonha"), para as quatro épocas de plantio, alcançado, em média, aos 100 dias após o plantio. Avaliaram-se as características de altura, diâmetro à altura do peito (DAPE), comprimento da espiga com (CECP) e sem palha (CESP), diâmetro da espiga com (DECP) e sem palha (DESP), massa fresca de espigas com (MECP) e sem palha (MESP) e produtividades total, referente à massa de espigas com palha (PTC), e comercial, referente ao total de espigas sem palha (PTS),

Tabela 1: Tratamentos utilizados e datas de implantação dos cultivos, solteiro e em consórcio, dos mangaritos 'Gigante' ou 'Pequeno', com milho verde, em cada experimento realizado

\begin{tabular}{|c|c|c|c|c|}
\hline \multirow{2}{*}{ Tratamento } & \multirow{2}{*}{$\begin{array}{c}\text { Descrição } \\
\text { dos Tratamentos }\end{array}$} & \multirow{2}{*}{$\begin{array}{c}\text { Mangarito } \\
\text { Data de plantio }\end{array}$} & \multirow{2}{*}{$\begin{array}{c}\text { Milho Verde } \\
\text { Data de plantio }\end{array}$} & \multirow{2}{*}{$(\mathbf{D A E})^{*}$} \\
\hline & & & & \\
\hline 1 & Mangarito Solteiro & $20 / 10 / 2015$ & - & - \\
\hline 2 & Mangarito + Milho Verde & $20 / 10 / 2015$ & $23 / 11 / 2015$ & 0 \\
\hline 3 & Mangarito + Milho Verde & $20 / 10 / 2015$ & $14 / 12 / 2015$ & 21 \\
\hline 4 & Mangarito + Milho Verde & $20 / 10 / 2015$ & 04/01/2016 & 42 \\
\hline 5 & Mangarito + Milho Verde & 20/10/2015 & 25/01/2016 & 63 \\
\hline 6 & Milho Verde Solteiro & - & $23 / 11 / 2015$ & 0 \\
\hline 7 & Milho Verde Solteiro & - & $14 / 12 / 2015$ & 21 \\
\hline 8 & Milho Verde Solteiro & - & 04/01/2016 & 42 \\
\hline 9 & Milho Verde Solteiro & - & 25/01/2016 & 63 \\
\hline
\end{tabular}

*DAE $=$ Dias após a emergência do mangarito 
ambas medidas em t.ha ${ }^{-1}$. Após colhidas as espigas verdes, as plantas de milho foram ceifadas rente ao solo e dispostas lateralmente na fileira de mangarito.

A colheita do mangarito foi realizada quando as plantas apresentavam em torno de $90 \%$ das folhas amareladas e secas, sintoma típico de senescência, o que aconteceu aos 250 dias após o plantio. Foram avaliadas a produtividade, em massa fresca, do cormo central (mãe) (CO) e das classes de cormelos grande, médio, pequeno e comerciais (somatório das três classes) e número de cormelos comerciais por planta (NCC). A classificação dos cormelos foi realizada com base no seu diâmetro, em peneiras de malhas, conforme Santin (2015), considerando-se comerciais o somatório das classes grande, médio e pequeno.

Os indicadores agroeconômicos empregados para avaliar a eficiência dos sistemas consorciados foram: o índice de Uso Eficiente da Terra (UET), renda bruta (RB), renda líquida (RL), vantagem monetária (VM) e vantagem monetária corrigida (VMc). O índice UET foi calculado conforme Willey (1979), obtido pela expressão: UET $=(\mathrm{Yab} / \mathrm{Yaa})$ $+(\mathrm{Yba} / \mathrm{Ybb})$, em que: Yab é a produtividade comercial da cultura "a" em consórcio com a cultura "b"; Yaa é a produtividade comercial da cultura "a" em monocultivo; Yba é a produtividade comercial da cultura "b" em consórcio com a cultura "a"; e Ybb é a produtividade comercial da cultura "b" em monocultivo.

A renda bruta (RB) foi calculada por meio da multiplicação da produtividade comercial da cultura em cada tratamento pelo preço do produto pago aos produtores, na CEASA-MG, em cada época de colheita. Para a cultura do mangarito, o preço do produto no dia da colheita foi de $\mathrm{R} \$$ $3,00 / \mathrm{kg}$ de cormelos comerciais e, para o milho verde, de $\mathrm{R} \$ 0,63 ; \mathrm{R} \$ 0,79 ; \mathrm{R} \$ 1,05 / \mathrm{kg}$ e de $\mathrm{R} \$ 0,94 / \mathrm{kg}$ de espigas comerciais, nas primeira, segunda, terceira e quarta épocas de plantio, respectivamente (CEASA-MG, 2016).

A renda líquida (RL) foi obtida pela subtração, da renda bruta, dos custos de produção derivados de insumos mais serviços. Esses custos foram calculados para cada tratamento, considerando-se os preços dos insumos e dos serviços utilizados nas culturas do mangarito e do milho verde, em nível experimental, com base nos indicadores de Gassi et al. (2014). A vantagem monetária (VM) e a vantagem monetária corrigida $(\mathrm{VMc})$ foram calculadas pelas seguintes expressões: $\mathrm{VM}=\mathrm{RB}(\mathrm{UET}-1) / \mathrm{UET}$ e VMc $=\mathrm{RL}$ (UET -1)/ UET, de acordo com Beltrão et al. (1984).

Os dados obtidos foram submetidos à análise de variância. As médias de produção do mangarito e os índices financeiros foram comparados entre si pelo teste de Tukey. Também foram realizados contrastes (teste F) entre cultivos solteiros e consorciados, para as características avaliadas no milho verde. Todos os testes foram a 5\% de probabilidade e as análises foram efetuadas com o programa estatístico Saeg 9.1 (UFV, 2007).

\section{RESULTADOS E DISCUSSÃO}

Quanto ao mangarito 'Gigante', as massas frescas de cormelos, das classes médio (CM) e pequeno (CP), e o número de cormelos comercializáveis por planta (NCC) não foram influenciados pelas épocas das associações do mangarito com o milho verde. Todavia, a associação do milho verde, realizada aos $42 \mathrm{DAE}$ do mangarito (T4), proporcionou massa fresca de cormo (CO), dentre todos os tratamentos, e de cormelos das classes grande e comercial, menor do que a do controle e a da associação aos 21 DAE (Tabela 2).

É possível que a menor produtividade de cormelos das classes grande e comercial, do tratamento de associação com milho aos $42 \mathrm{DAE}$, possa ter ocorrido em virtude de seu crescimento lento, na fase inicial, e do crescimento rápido das plantas de milho, o que promoveu maior competição por fatores de crescimento, em especial, por luz, interferindo significativamente na expressão do potencial produtivo das plantas de mangarito "Gigante". O sombreamento reduz a densidade do fluxo de luz, limitando as taxas fotossintética e de evapotranspiração (Lopes \& Lima, 2015). O mangarito 'Pequeno' apresentou comportamento um pouco diferente do mangarito 'Gigante', não havendo diferença entre tratamentos quanto à produção de cormo e de cormelos da classe pequeno. Porém, houve diferença para produção de cormelos das classes grande, médio e comercial. A associação do milho verde com mangarito 'Pequeno', aos $21 \mathrm{DAE}$, proporcionou menor produção de cormelos da classe grande, em comparação com a do controle e com a da associação ao 0 DAE, e menor produção de cormelos comerciais que a do controle. Por sua vez, a associação aos 63 DAE proporcionou menor produção de cormelos médios, não diferindo da associação ao 0 DAE, e menor produção de cormelos comerciais que a do controle, não diferindo da associação aos 21 DAE (Tabela 2).

Não somente as espécies consorciadas, mas o momento do ciclo em que são consorciadas, são fundamentais para o sucesso dessa prática. Vieira et al. (2014) obtiveram maiores rendimentos da cultura do taro quando a associação com o feijão-vagem foi realizada aos 21 e 42 DAP. Todavia, o desempenho do feijão-vagem foi fortemente afetado pela competição com as plantas de taro nesses períodos. Embora da mesma família do mangarito, o taro é uma planta muito mais exuberante e competitiva que o mangarito e o feijão-vagem, por sua vez, muito menos competitivo que o milho. Portanto, o mangarito ficou em grande desvantagem competitiva com o milho.

Ambas as variedades de mangarito, 'Gigante' e 'Pequeno', apresentaram, numericamente, maiores produções de cormelos comerciais no cultivo solteiro. O milho, por ser uma espécie de metabolismo $\mathrm{C}_{4}$ e com taxa de cresci- 
mento elevada, cresceu rapidamente e causou certa restrição de luz às plantas de mangarito. Outro fator que contribuiu para o pior desempenho do mangarito nos consórcios foi a injúria sofrida pelas plantas de mangarito após o corte das plantas de milho. Dependendo da época em que as plantas de milho foram cortadas, após colheita das espigas, as folhas de mangarito, por estarem sob o dossel do milho, ao serem expostas bruscamente à radiação solar intensa do verão, sofreram solarização (branqueamento por degradação dos cloroplastos), com redução da área fotossintética, fato que gerou gasto energético extra pelas plantas para a recuperação.

Todavia, mesmo nos tratamentos em consórcio, as produtividades obtidas com ambas as variedades de mangarito, neste trabalho, foram bem superiores às encontradas por Castro et al. (2017), Heredia Zárate et al. (2005) e Gassi et al. (2014), avaliando massa de cormelo-semente e espaçamento. Isso mostra que, agronomicamente, pode-se considerar viável a consorciação do mangarito com milho verde, desde que se acrescentem determinados cuidados à colheita, especialmente para evitar injúrias decorrentes do revolvimento do solo na semeadura do milho.

Em relação à cultura do milho, tanto as características de crescimento das plantas como as produtivas (massa fresca de espigas com e sem palha) não diferiram entre tratamentos solteiros e consorciados, nas respectivas épocas de associação e em ambas as variedades de mangarito estudadas (Tabela 3). Esses resultados evidenciam que as plantas de milho não sofreram competição das plantas de mangarito pelos fatores de crescimento, mesmo quando a associação ocorreu aos 63 DAE. Como abordado anterior- mente, o milho, por ser uma espécie que tem crescimento rápido, tornou-se dominante, quando no consórcio, não sofrendo nenhuma interferência das plantas de mangarito.

Na Tabela 4, encontram-se os indicadores econômicos dos sistemas consorciados dos mangaritos 'Gigante' e 'Pequeno' com milho verde. Os sistemas consorciados proporcionaram maior aproveitamento do fator terra do que o sistema solteiro, uma vez que os índices de uso da terra (UET) foram maiores que a unidade. No mangarito 'Gigante', o UET variou de 107\%, no consórcio com milho verde aos 63 DAE, a 134\%, no consórcio ao 0 DAE. Para o mangarito 'Pequeno', o UET variou de $85 \%$, no consórcio aos $42 \mathrm{DAE}$, a 156\%, aos $21 \mathrm{DAE}$. Isso significa que seriam necessárias essas percentagens a mais, em área física, para que as culturas do milho verde e mangarito, em plantios solteiros, produzissem o equivalente à produção consorciada em um hectare.

Apesar do UET elevado, no consórcio estabelecido ao 0 DAE para o mangarito 'Gigante', essa época da associação foi a que proporcionou menor renda bruta que a da associação estabelecida aos 21 DAE e menor renda líquida que a do controle e também que a da associação estabelecida aos 21 DAE. Valor de UET elevado indica o melhor uso da terra para produção de alimento; todavia, neste trabalho, o mangarito é mais valorizado e, quando em associação com milho, teve a produtividade afetada (Tabela 2), o que resultou em menor renda. Por sua vez, as vantagens monetárias (VM e VMc) dos consórcios não apresentaram diferenças estatísticas, porém, o maior valor foi observado também no consórcio estabelecido aos 21 DAE.

Tabela 2: Rendimentos médios de massa fresca de Cormo (CO) e de Cormelos grandes (CG), médios (CM), pequenos (CP), comerciais (CC) e número de Cormelos comerciais por planta (NCC), de mangaritos 'Gigante' (Mg) e 'Pequeno' (Mp), em monocultura e em consórcio com milho (Mi), nas épocas de associação das culturas

\begin{tabular}{|c|c|c|c|c|c|c|}
\hline \multirow{2}{*}{ Tratamentos } & $\mathrm{CO}$ & CG & $\mathbf{C M}$ & $\mathbf{C P}$ & $\mathrm{CC}$ & NCC \\
\hline & \multicolumn{5}{|c|}{ t ha $^{-1}$} & unidade $\mathbf{~ p l}^{-1}$ \\
\hline \multicolumn{7}{|c|}{ Mangarito 'Gigante' } \\
\hline 1-Mg solteiro & $4,12 a^{*}$ & $14,21 \mathrm{a}$ & $5,26 \mathrm{a}$ & 1,01 & $20,48 \mathrm{a}$ & 71,10 \\
\hline $2-\mathrm{Mg}+\mathrm{Mi}(0 \mathrm{DAE})$ & $3,50 \mathrm{a}$ & $11,98 \mathrm{ab}$ & $3,53 \mathrm{a}$ & 0,57 & $16,09 \mathrm{bc}$ & 40,02 \\
\hline 3-Mg + Mi (21 DAE) & $3,76 \mathrm{a}$ & $13,89 \mathrm{a}$ & $5,06 \mathrm{a}$ & 0,71 & $19,67 \mathrm{ab}$ & 50,10 \\
\hline 4-Mg + Mi (42 DAE) & $2,52 \mathrm{~b}$ & $9,5 \mathrm{~b}$ & $5,15 \mathrm{a}$ & 0,87 & $15,61 \mathrm{c}$ & 58,95 \\
\hline 5-Mg + Mi (63 DAE) & $3,54 \mathrm{a}$ & $11,79 \mathrm{ab}$ & $4,69 \mathrm{a}$ & 0,80 & $17,29 \mathrm{abc}$ & 60,07 \\
\hline $\mathrm{CV}(\%)$ & 8,51 & 9,93 & 17,78 & 26,79 & 8,36 & 27,88 \\
\hline \multicolumn{7}{|c|}{ Mangarito 'Pequeno' } \\
\hline 1-Mp solteiro & $2,83 *$ & $9,48 \mathrm{a}$ & $3,74 \mathrm{a}$ & $0,76 \mathrm{a}$ & $13,98 \mathrm{a}$ & 44,37 \\
\hline 2-Mp + Mi (0 DAE) & 2,51 & $9,18 \mathrm{a}$ & $2,19 \mathrm{~b}$ & $0,59 \mathrm{a}$ & $11,96 \mathrm{ab}$ & 36,82 \\
\hline 3-Mp + Mi (21 DAE) & 2,43 & $4,29 \mathrm{~b}$ & $3,45 \mathrm{a}$ & $0,59 \mathrm{a}$ & $8,34 \mathrm{~b}$ & 36,17 \\
\hline 4-Mp + Mi (42 DAE) & 2,35 & $6,34 \mathrm{ab}$ & $3,82 \mathrm{a}$ & $0,77 \mathrm{a}$ & $10,94 \mathrm{ab}$ & 41,67 \\
\hline 5-Mp + Mi (63 DAE) & 2,48 & $6,33 \mathrm{ab}$ & $2,35 \mathrm{~b}$ & $0,70 \mathrm{a}$ & $9,38 \mathrm{~b}$ & 36,60 \\
\hline$\overline{\mathrm{CV}(\%)}$ & 29,28 & 14,36 & 13,51 & 32,46 & 18,42 & 31,34 \\
\hline
\end{tabular}

* Médias, nas colunas, seguidas por pelo menos uma mesma letra, não diferem entre si pelo teste de Tukey, a $5 \%$ de probabilidade. 
Tabela 3: Valores médios, observados em milho verde, dos contrastes entre os cultivos solteiros e consorciados com mangaritos 'Gigante' e 'Pequeno' (v1, v2, v3 e v4), nas quatro épocas de associação, das características altura (ALT), diâmetro à altura do peito (DAPE), comprimento da espiga com (CECP) e sem palha (CESP), diâmetro da espiga com (DECP) e sem palha (DESP), massa fresca de espiga com (MECP) e sem palha (MESP), produtividades total e comercial de espigas com (PTC) e sem palha (PTS), respectivamente

\begin{tabular}{|c|c|c|c|c|c|c|c|c|c|c|c|}
\hline \multirow{2}{*}{ Contrastes } & \multirow{2}{*}{ Trat. } & \multirow{2}{*}{$\begin{array}{r}\text { ALT } \\
(\mathbf{m})\end{array}$} & DAPE & CECP & \multirow{2}{*}{$\begin{array}{c}\text { CESP } \\
(\mathrm{cm})\end{array}$} & DRCP & \multirow[t]{2}{*}{ DESP } & МECP & MESP & PTC & PTS \\
\hline & & & & & & & & \multicolumn{2}{|c|}{ (g) } & \multicolumn{2}{|c|}{$\left(\right.$ t.ha $\left.{ }^{-1}\right)$} \\
\hline \multicolumn{12}{|c|}{ Mangarito 'Gigante' } \\
\hline \multirow[b]{2}{*}{$\hat{\mathrm{Y}} 1$} & 6 & 2,81 & 2,58 & 32,03 & 22,15 & 5,51 & 4,43 & 427,75 & 272,75 & 14,25 & 9,09 \\
\hline & 2 & $\begin{array}{l}2,88 \\
-0,07^{\mathrm{NS}^{*}}\end{array}$ & $\begin{array}{l}2,35 \\
0,13 \text { NS }\end{array}$ & $\begin{array}{l}32,75 \\
-0,72^{\mathrm{NS}}\end{array}$ & $\begin{array}{c}20,94 \\
1,51^{\mathrm{Ns}}\end{array}$ & $\begin{array}{c}5,87 \\
-0,36^{\text {NS }}\end{array}$ & $\begin{array}{l}4,33 \\
0,10^{\mathrm{NS}}\end{array}$ & $\begin{array}{l}446,93 \\
-19,18^{\text {NS }}\end{array}$ & $\begin{array}{c}260,00 \\
12,75^{\text {NS }}\end{array}$ & $\begin{array}{l}14,89 \\
-0,64^{\mathrm{NS}}\end{array}$ & $\begin{array}{l}8,66 \\
0,43 \text { Ns }\end{array}$ \\
\hline \multirow[b]{2}{*}{$\hat{\mathrm{Y}} 2$} & 7 & 2,76 & 1,76 & 32,52 & 22,15 & 5,80 & 4,49 & 402,68 & 256,90 & 13,42 & 8,56 \\
\hline & 3 & $\begin{array}{l}2,63 \\
0,13^{\mathrm{NS}}\end{array}$ & $\begin{array}{c}1,85 \\
-0,09^{\mathrm{NS}}\end{array}$ & $\begin{array}{l}33,62 \\
-1,10^{\mathrm{NS}}\end{array}$ & $\begin{array}{l}22,65 \\
-0,50^{\mathrm{NS}}\end{array}$ & $\begin{array}{l}5,37 \\
0,43^{\mathrm{NS}}\end{array}$ & $\begin{array}{l}4,01 \\
0,48^{\mathrm{NS}}\end{array}$ & $\begin{array}{c}351,12 \\
51,56^{\mathrm{NS}}\end{array}$ & $\begin{array}{c}193,81 \\
63,09^{\text {NS }}\end{array}$ & $\begin{array}{c}11,70 \\
1,72^{\text {NS }}\end{array}$ & $\begin{array}{l}6,46 \\
2,10^{\mathrm{NS}}\end{array}$ \\
\hline \multirow{3}{*}{$\widehat{\mathrm{Y}} 3$} & 8 & 2,62 & 1,29 & 31,24 & 20,31 & 5,12 & 4,14 & 368,75 & 187,64 & 12,90 & 8,70 \\
\hline & 4 & $\begin{array}{c}2,67 \\
-0,05^{\mathrm{NS}}\end{array}$ & $\begin{array}{c}1,58 \\
-0,29^{\mathrm{NS}}\end{array}$ & $\begin{array}{l}31,45 \\
-0,21^{\mathrm{NS}}\end{array}$ & $\begin{array}{l}20,98 \\
-0,67^{\mathrm{NS}}\end{array}$ & $\begin{array}{c}5,34 \\
-0,22^{\text {NS }}\end{array}$ & $\begin{array}{c}4,22 \\
-0,08 \text { NS }\end{array}$ & $\begin{array}{l}397,93 \\
-29,18^{\mathrm{NS}}\end{array}$ & $\begin{array}{l}223,55 \\
-36,51^{\mathrm{NS}}\end{array}$ & $\begin{array}{l}13,43 \\
-0,53^{\mathrm{NS}}\end{array}$ & $\begin{array}{l}8,53 \\
0,17^{\mathrm{NS}}\end{array}$ \\
\hline & 9 & 2,43 & 1,33 & 31,62 & 20,68 & 5,15 & 4,02 & 372,50 & 225,81 & 12,41 & 7,52 \\
\hline$\hat{\mathrm{Y}} 4$ & 5 & $\begin{array}{c}2,48 \\
-0,05^{\mathrm{NS}}\end{array}$ & $\begin{array}{l}1,32 \\
0,011^{\mathrm{NS}}\end{array}$ & $\begin{array}{l}32,21 \\
-0,59^{\mathrm{NS}}\end{array}$ & $\begin{array}{l}24,94 \\
-4,26^{\mathrm{NS}}\end{array}$ & $\begin{array}{c}5,41 \\
-0,26^{\mathrm{NS}} \\
\end{array}$ & $\begin{array}{c}4,16 \\
-0,14^{\mathrm{NS}}\end{array}$ & $\begin{array}{c}351,65 \\
20,85^{\mathrm{NS}}\end{array}$ & $\begin{array}{c}197,43 \\
28,38^{\mathrm{NS}}\end{array}$ & $\begin{array}{c}11,72 \\
0,69^{\mathrm{NS}}\end{array}$ & $\begin{array}{l}6,58 \\
0,94^{\mathrm{NS}}\end{array}$ \\
\hline \multicolumn{12}{|c|}{ Mangarito 'Pequeno' } \\
\hline \multirow[b]{2}{*}{$\hat{Y} 1$} & 6 & 2,73 & 1,23 & 31,62 & 21,41 & 2,73 & 2,2 & 459,43 & 277,31 & 15,31 & 9,24 \\
\hline & 2 & $\begin{array}{l}2,72 \\
0,01^{\text {NS* }^{*}}\end{array}$ & $\begin{array}{l}1,17 \\
0,06^{\mathrm{NS}}\end{array}$ & $\begin{array}{l}31,94 \\
-0,32^{\mathrm{NS}}\end{array}$ & $\begin{array}{c}21,25 \\
0,16^{\mathrm{NS}}\end{array}$ & $\begin{array}{l}2,72 \\
0,01^{\mathrm{NS}}\end{array}$ & $\begin{array}{l}2,1 \\
0,01^{\mathrm{NS}}\end{array}$ & $\begin{array}{c}422,06 \\
37,37^{\mathrm{NS}}\end{array}$ & $\begin{array}{c}250,50 \\
26,81^{\mathrm{NS}}\end{array}$ & $\begin{array}{c}14,06 \\
1,25^{\mathrm{NS}}\end{array}$ & $\begin{array}{l}8,35 \\
0,89^{\text {NS }}\end{array}$ \\
\hline \multirow[b]{2}{*}{$\hat{\mathrm{Y}} 2$} & 7 & 2,85 & 1,25 & 30,79 & 21,59 & 2,85 & 1,59 & 369,62 & 229,56 & 12,32 & 7,66 \\
\hline & 3 & $\begin{array}{l}2,48 \\
0,37^{\mathrm{NS}}\end{array}$ & $\begin{array}{l}1,02 \\
0,23^{\mathrm{NS}}\end{array}$ & $\begin{array}{l}32,12 \\
-1,33^{\mathrm{NS}}\end{array}$ & $\begin{array}{c}21,68 \\
0,09^{\mathrm{NS}}\end{array}$ & $\begin{array}{l}2,48 \\
0,37^{\mathrm{NS}}\end{array}$ & $\begin{array}{c}1,71 \\
-0,12^{\mathrm{NS}}\end{array}$ & $\begin{array}{c}358,12 \\
11,5^{\mathrm{NS}}\end{array}$ & $\begin{array}{c}211,68 \\
17,88^{\mathrm{NS}}\end{array}$ & $\begin{array}{c}11,93 \\
0,39^{\mathrm{NS}}\end{array}$ & $\begin{array}{l}7,03 \\
0,63^{\mathrm{NS}}\end{array}$ \\
\hline \multirow[b]{2}{*}{$\hat{Y} 3$} & 8 & 2,67 & 1,39 & 31,97 & 21,87 & 2,67 & 1,64 & 367,00 & 209,33 & 11,93 & 6,98 \\
\hline & 4 & $\begin{array}{l}2,24 \\
0,43^{\mathrm{NS}}\end{array}$ & $\begin{array}{l}1,14 \\
0,25^{\mathrm{NS}}\end{array}$ & $\begin{array}{l}32,76 \\
-0,79^{\mathrm{NS}}\end{array}$ & $\begin{array}{l}22,67 \\
-0,8^{\mathrm{NS}}\end{array}$ & $\begin{array}{l}2,24 \\
0,43^{\mathrm{NS}}\end{array}$ & $\begin{array}{c}1,87 \\
-0,23^{\mathrm{NS}}\end{array}$ & $\begin{array}{c}347,12 \\
19,13^{\mathrm{NS}}\end{array}$ & $\begin{array}{c}199,96 \\
9,37^{\mathrm{NS}}\end{array}$ & $\begin{array}{c}11,57 \\
0,36^{\mathrm{NS}}\end{array}$ & $\begin{array}{l}6,66 \\
0,32^{\mathrm{NS}}\end{array}$ \\
\hline \multirow[b]{2}{*}{$\hat{\mathrm{Y}} 4$} & 9 & 2,43 & 1,33 & 31,56 & 21,37 & 2,43 & 0,98 & 358,26 & 230,12 & 11,94 & 7,67 \\
\hline & 5 & $\begin{array}{c}2,52 \\
-0,09^{\mathrm{NS}}\end{array}$ & $\begin{array}{l}1,04 \\
0,29^{\mathrm{NS}}\end{array}$ & $\begin{array}{l}32,39 \\
-0,83^{\text {NS }}\end{array}$ & $\begin{array}{l}22,28 \\
-0,91^{\mathrm{NS}}\end{array}$ & $\begin{array}{c}2,52 \\
-0,09^{\mathrm{NS}}\end{array}$ & $\begin{array}{c}1,73 \\
-1,75^{\mathrm{NS}}\end{array}$ & $\begin{array}{c}357,87 \\
0,39^{\mathrm{NS}}\end{array}$ & $\begin{array}{c}219,98 \\
10,14^{\mathrm{NS}}\end{array}$ & $\begin{array}{l}11.93 \\
0,01^{\mathrm{NS}}\end{array}$ & $\begin{array}{l}\quad 7,33 \\
0.34^{\mathrm{NS}}\end{array}$ \\
\hline
\end{tabular}

*NS: Não significativo pelo teste F a 5\% de probabilidade. v1 - Milho verde solteiro a 0 DAE (T6) vs. Milho verde consorciado com o mangarito ao 0 DAE (T2); v2 - Milho verde solteiro aos 21 DAE (T7) vs. Milho verde consorciado com o mangarito aos 21 DAE (T3); v3 - Milho verde solteiro aos 42 DAE (T8) vs. Milho verde consorciado com mangarito aos 42 DAE (T4); v4 - Milho verde solteiro aos 60 DAE (T9) vs. Milho verde consorciado com o mangarito aos 63 DAE (T5). 
A produtividade de cormelos comerciais do mangarito 'Gigante' em consórcio com milho verde, aos 21 e aos 63 DAE, não diferiu da produtividade do cultivo solteiro (20,48 contra 19,67 e 17,29 t ha $^{-1}$ ), fato que justifica o valor aproximado de renda líquida desses tratamentos, uma vez que a rentabilidade obtida com a cultura do milho não foi tão compensatória (Tabela 2). Assim, como observado com o mangarito 'Gigante', para o mangarito 'Pequeno', o UET elevado obtido nos consórcios estabelecidos aos 21 e 63 DAE não correspondeu, em termos de rendas bruta e líquida, ficando abaixo do controle (Tabela 4). Isso é explicado pela maior produção de mangarito no sistema solteiro e sua maior valorização, em associação com o milho verde (Tabela 2).

Embora o consórcio do mangarito 'Gigante' com milho verde, aos $21 \mathrm{DAE}$, tenha sido mais vantajoso, em termos econômicos, sobretudo em relação ao consórcio estabelecido, ao 0 DAE, o mesmo não foi observado com o mangarito 'Pequeno'. Para este ('Pequeno'), não houve diferença entre as épocas de associação dos consórcios, quanto às rendas bruta e líquida, mas os consórcios estabelecidos aos 21 e aos 63 DAE apresentaram menores rendas que as do controle. Assim como para o 'Gigante', a produção de cormelos comerciais do mangarito 'Pequeno', no cultivo solteiro, foi muito superior aos consórcios estabelecidos aos 21 e 63 DAE (Tabela 2), o que refletiu diretamente na rentabilidade (Tabela 4).

Diferentemente do 'Gigante', para o mangarito "Pequeno" as épocas dos consórcios apresentaram diferenças quanto às vantagens monetárias, com o consórcio estabelecido ao 0 DAE sobressaindo-se em termos de VM e VMc, em relação aos das demais épocas de associação. As VM e VMc são dependentes das rendas (bruta e líquida, respectivamente), mas também do UET. Assim sendo, para um mesmo UET, maiores rendas resultam em maiores VMe VMc, mas para um mesmo valor de renda, UET maior resulta em maior VM ou VMc, uma vez que necessitaria de menor área física para obter a mesma renda.

Embora presuma-se que existam grandes possibilidades da obtenção de maiores lucros quando as culturas estão em associação, vários fatores podem contribuir para que nem sempre isso ocorra. Todavia, neste trabalho, embora os tratamentos em consórcio não se tenham sobressaído em termos econômicos, em comparação com o do cultivo do mangarito solteiro, vale ressaltar que as espigas de milho tiveram iniciadas as colheitas com cerca de 100 dias da semeadura do milho (133 dias do plantio do mangarito na primeira época da associação), ou seja, há uma antecipação de entrada de recursos financeiros para o agricultor, o que é desejável.

Tabela 4: Índice de Uso da Terra (UET), Renda Bruta (RB), Renda Líquida (RL), Vantagem Monetária (VM) e Vantagem Monetária corrigida (VMc) dos tratamentos dos mangaritos 'Gigante' (Mg) e 'Pequeno' (Mp), solteiros e em associação com milho verde (Mi)

\begin{tabular}{|c|c|c|c|c|c|}
\hline \multirow{2}{*}{ Tratamentos } & UET & $\mathbf{R B}\left(\mathbf{R} \$ \mathbf{h a}^{-1}\right)$ & $\mathbf{R L}\left(\mathbf{R} \$ \mathbf{h a}^{-1}\right)$ & VM $\left(\mathbf{R} \$\right.$ ha $\left.^{-1}\right)$ & $\operatorname{VMc}\left(\mathbf{R} \$ \mathrm{ha}^{-1}\right)$ \\
\hline & \multicolumn{5}{|c|}{ Mangarito 'Gigante' } \\
\hline 1- Mg solteiro & $1,00 \mathrm{c}^{*}$ & $61.449,38 \mathrm{ab}$ & $53.151,38 \mathrm{a}$ & - & - \\
\hline 2- $\mathrm{Mg}+\mathrm{Mi}(0 \mathrm{DAE})$ & $2,34 \mathrm{a}$ & $53.734,46 \mathrm{~b}$ & $41.355,46 \mathrm{c}$ & $30.776,08$ a & $23.691,83 \mathrm{a}$ \\
\hline 3- $\mathrm{Mg}+\mathrm{Mi}$ (21 DAE) & $2,15 \mathrm{ab}$ & $64.128,08 \mathrm{a}$ & $51.749,08 \mathrm{ab}$ & $34.223,61 \mathrm{a}$ & $27.616,26 \mathrm{a}$ \\
\hline 4- Mg + Mi (42 DAE) & $2,28 \mathrm{ab}$ & $55.721,03 \mathrm{ab}$ & $43.402,03 \mathrm{bc}$ & $31.308,12 \mathrm{a}$ & $24.356,88 \mathrm{a}$ \\
\hline 5- $\mathrm{Mg}+\mathrm{Mi}(63 \mathrm{DAE})$ & $2,07 \mathrm{~b}$ & $58.060,79 \mathrm{ab}$ & $45.681,79 \mathrm{abc}$ & $30.017,09 \mathrm{a}$ & $23.636,01 \mathrm{a}$ \\
\hline 6- Mi solteiro (0 DAE) & $1,00 \mathrm{c}$ & $5.727,69 \mathrm{c}$ & $1.645,77 \mathrm{~d}$ & - & - \\
\hline 7- Mi solteiro (21 DAE) & $1,00 \mathrm{c}$ & $6.764,96 \mathrm{c}$ & $2.683,04 \mathrm{~d}$ & - & - \\
\hline 8- Mi solteiro (42 DAE) & $1,00 \mathrm{c}$ & $9.135,00 \mathrm{c}$ & $5.053,08 \mathrm{~d}$ & - & - \\
\hline 9- Mi solteiro (63 DAE) & $1,00 \mathrm{c}$ & $7.075,38 \mathrm{c}$ & $2.993,46 \mathrm{~d}$ & - & - \\
\hline \multirow[t]{2}{*}{$\mathrm{CV}(\%)$} & 5,89 & 9,71 & 12,62 & 7,78 & 9,00 \\
\hline & \multicolumn{5}{|c|}{ Mangarito 'Pequeno' } \\
\hline 1- Mp solteiro & $1,00 \mathrm{c}^{*}$ & $41.949,58 \mathrm{a}$ & $33.651,00 \mathrm{a}$ & - & - \\
\hline 2- Mp + Mi (0 DAE) & $2,10 \mathrm{ab}$ & $35.899,64 \mathrm{ab}$ & $23.520,12 \mathrm{ab}$ & $18.058,90 \mathrm{a}$ & $11.813,06 \mathrm{a}$ \\
\hline 3- Mp + Mi (21 DAE) & $2,56 \mathrm{a}$ & $25.019,74 \mathrm{~b}$ & $12.640,76 \mathrm{~b}$ & $15.129,63 \mathrm{~b}$ & $7.612,03 \mathrm{~b}$ \\
\hline 4- Mp + Mi (42 DAE) & $1,85 \mathrm{~b}$ & $32.822,17 \mathrm{ab}$ & $20.443,17 \mathrm{ab}$ & $14.535,23 \mathrm{~b}$ & $8.895,20 \mathrm{~b}$ \\
\hline 5- Mp + Mi (63 DAE) & $2,13 \mathrm{ab}$ & $28.149,71 \mathrm{~b}$ & $15.770,22 \mathrm{~b}$ & $14.594,06 \mathrm{~b}$ & $8.138,00 \mathrm{~b}$ \\
\hline 6- Mi solteiro (0 DAE) & $1,00 \mathrm{c}$ & $5.260,50 \mathrm{c}$ & $1.178,58 \mathrm{c}$ & - & - \\
\hline 7- Mi solteiro (21 DAE) & $1,00 \mathrm{c}$ & $6.043,50 \mathrm{c}$ & $1.961,58 \mathrm{c}$ & - & - \\
\hline 8- Mi solteiro (42 DAE) & $1,00 \mathrm{c}$ & $7.329,00 \mathrm{c}$ & $3.247,08 \mathrm{c}$ & - & - \\
\hline 9- Mi solteiro (63 DAE) & $1,00 \mathrm{c}$ & $6.857,30 \mathrm{c}$ & $2.775,38 \mathrm{c}$ & - & - \\
\hline $\mathrm{CV}(\%)$ & 15,04 & 25,33 & 28,47 & 8,32 & 15,96 \\
\hline
\end{tabular}

* Médias, nas colunas, seguidas por pelo menos uma mesma letra não diferem entre si pelo teste de Tukey a 5\% de probabilidade. 
Em se tratando de cultivo consorciado, a rentabilidade pode ser muito variável (Brito, 2017). As culturas consorciadas, em termos de fenologia, tolerância à competição pelos fatores de crescimento, época das associações, arranjos e manejo utilizados e, finalmente, a quantidade e o valor do produto colhido, são fatores que influenciarão nos resultados financeiros. Contudo, neste trabalho, os indicadores econômicos apresentaram-se mais vantajosos, em comparação com os de outros trabalhos. Assim, Castro et al. (2017) obtiveram maior renda líquida ( $\mathrm{R} \$ 5.600,14 / \mathrm{ha})$, com aplicação de cama de frango com base de casca de arroz e espaçamento reduzido (12,5 cm entre plantas). Heredia Zárate et al. (2013) obtiveram maior renda líquida ( $\mathrm{R} \$ 2.860,48 / \mathrm{ha}$ ), no cultivo do mangarito com quatro fileiras de plantas e com duas amontoas e, Gassi et al. (2014), maior renda líquida ( $\mathrm{R} \$ 7.712,56 / \mathrm{ha})$, com mudas de 5,52 g no espaçamento de $0,15 \mathrm{~m}$.

De acordo com o "princípio da exclusão competitiva", descrito por Vandermeer (1990), quando duas espécies têm requerimentos distintos, competem entre si fracamente, sobrevivendo indefinidamente no mesmo ecossistema; porém, quando os requerimentos são similares, competem entre si fortemente, com uma delas tendendo a se extinguir em dado período de tempo. Neste trabalho, apesar do consórcio ter provocado impacto sobre o desempenho das variedades de mangarito, a associação do milho com mangarito não inviabilizou a obtenção de cormelos comerciais do mangarito, evidenciando que, de certa forma, a competição entre as culturas foi fraca, talvez pelo curto período de convivência das duas culturas devido ao ciclo do milho (100 dias).

\section{CONCLUSÕES}

Apesar de os cultivos solteiros apresentarem maiores produtividades, as consorciações dos mangaritos 'Gigante' e 'Pequeno' com milho verde é viável por apresentarem UET acima da unidade, em todos os consórcios estudados.

Em termos econômicos, a associação do milho verde com mangarito 'Gigante' deve ser instalada, preferencialmente, aos 21 dias após a emergência das plantas do mangarito e, para o mangarito 'Pequeno', ao 0 (zero) dia.

\section{REFERÊNCIAS}

Beltrão NEM, Nóbrega LB, Azevêdo DMP \& Vieira DJ (1984) Comparação entre indicadores agroeconômicos de avaliação de agroecossistemas consorciados e solteiros envolvendo algodão upland e feijão "caupi". Campina Grande, CNPA. 21p. (Boletim de Pesquisa, 15).

Brasil - Ministério da Agricultura, Pecuária e Abastecimento (2013) Manual de hortaliças não-convencionais. Brasília, MAPA/ACS. $99 \mathrm{p}$

Brito AU (2017) Viabilidade agroeconômica da consorciação do taro com outras hortaliças. Tese de Doutorado. Universidade Federal de Viçosa, Viçosa. 60p.
Brito AU, Puiatti M, Cecon PR, Finger FL \& Mendes TCD (2017) Viabilidade agroeconômica dos consórcios taro com brócolis, couve-chinesa, berinjela, jiló, pimentão e maxixe. Revista Brasileira de Ciências Agrárias, 12:296-302.

Castro LFQ, Heredia Zárate NA, Vieira MC, Torales EP \& Luqui LL (2017) Produtividade e rentabilidade do mangarito sob diferentes densidades de plantio e fontes de resíduo orgânico. Revista Scientia Agrária, 18:107-115.

CATI - Coordenadoria de Assistência Técnica Integral (2018) Milho Al Bandeirante. Disponível em: <https://biodinamica.org.br/images/pdf/milhoorganico/milho-al-bandeirante.pdf. $\geq$. Acessado em: 31 de janeiro de 2018.

Cavalcanti TFM (2011) Aspectos morfológicos, agronômicos e nutricionais de acessos de mangarito. Dissertação de Mestrado. Montes Claros, ICA/UFMG. 65p.

CEASA/MG - Centrais de abastecimento de Minas Gerais (2016) Disponível em: <http://200.198.51.69/detec/ofertas_medio_ prd/ofertas_medio_prd.php.>. Acessado em: 23 de novembro de 2017.

Cecílio Filho AB, Rezende BLA, Barbosa JC, Feltrim AL, Silva GS \& Grangeiro LC (2008) Interação entre alface e tomateiro consorciados em ambiente protegido, em diferentes épocas. Horticultura Brasileira 26:158-164.

Costa CA, Ramos SJ, Alves DS, Fernandes LA, Sampaio RA \& Martins ER (2008) Nutrição mineral do mangarito num Latossolo Vermelho Amarelo. Horticultura Brasileira, 26:102-106.

Embrapa - Empresa Brasileira de Pesquisa Agropecuária (2013) Sistema brasileiro de classificação de solos. $3^{a}$ ed. Brasília, Embrapa. 353p.

Embrapa (2009) Empresa Brasileira de Pesquisa Agropecuária. Manual de análises químicas de solos, plantas e fertilizantes. $2^{\text {a }}$ ed. Brasília, Informação Tecnológica. 628p.

Gassi RS, Heredia Zárate NA, Vieira MC \& Torales EP (2014) Desempenho agroecônomico do mangarito 'Comum' cultivado com espaçamentos entre plantas e massas de rizomas-semente. Semina: Ciências Agrárias, 35:149-160.

Gliessman SR (2009) Agroecologia: processos ecológicos em agricultura sustentável. 4a ed. Porto Alegre, UFRGS. 656p.

Gonçalves EG (2011) The commonly cultivated species of Xanthosoma Schott (Araceae), including four new species. Aroideana, 34:02-23.

Heredia Zárate NA, Vieira MC \& Pontim BCA (2005) Arranjo de plantas na produção do mangarito (Xanthosoma mafaffa Schott) 'Comum'. Acta Scientiarum Agronomy, 27:409-413.

Heredia Zárate NA, Vieira MC, Helmich M \& Maria MA (2006) Tamanho de rizomas-semente e fileiras de plantas no canteiro na produção do mangarito cv. Comum. Ciência e Agrotecnologia, 30:907-913.

Heredia Zárate NA, Sangalli CMS, Vieira MC, Jorge RPG, Torales EP \& Salles NA (2013) Produção agroeconômica do mangarito submetido a diferentes arranjos espaciais e tratos culturais. Horticultura Brasileira, 31:476-482.

Kinupp VK \& Lorenzi H (2014) Plantas Alimentícias Não Convencionais (PANC) no Brasil: Guia de identificação, aspectos nutricionais e receitas ilustradas. São Paulo, Instituto Plantarum de Estudos da Flora. 768p.

Köppen W (1948) Climatologia: con un estudio de los climas de la tierra. México, Fondo de Cultura Econômica. 479p.

Lopes NF \& Lima MGS (2015) Fisiologia da Produção. Viçosa, Editora UFV. 429p. 
Ribeiro CA, Guimarães PTG \& Alvares VH (1999) Recomendações para o uso de corretivos e fertilizantes em Minas Gerais $5^{\text {a }}$ Aproximação. Viçosa, CFSEMG. 359p.

Rocha DR (2008) Desempenho de cultivares de milho verde submetidas a diferentes populações de plantas em condições de irrigação. Tese de Doutorado. Universidade Estadual Paulista, Jaboticabal. 89p.

Universidade Federal de Viçosa (2007) SAEG: Sistema para Análises Estatísticas e Genéticas. Versão 9.1. Viçosa, Fundação Arthur Bernardes. CD-ROM.

Santin CR (2015) Crescimento e produção do mangarito sob as malhas termorrefletora, difusora e sombrite. Dissertação Mestrado. Universidade Federal de Viçosa, Viçosa. 51p.
Taiz L \& Zeiger E (2013) Fisiologia Vegetal. Porto Alegre, Artmed. $954 \mathrm{p}$.

Vandermeer JH (1990) Intercropping. In: Gliessman SR (Ed.) Agroecology: researching the ecological basis for sustainable agriculture. New York, Springer-Verlag. p.481-516.

Vieira JCB, Puiatti M, Cecon PR, Bhering AS, Silva GCC \& Colombo JN (2014) Viabilidade agroeconômica da consorciação do taro com feijão-vagem indeterminado em razão da época de plantio. Revista Ceres, 61:229-233.

Willey RW (1979) Intercropping - its importance and research needs. Part 1. Competition and yield advantages. Field Crop Abstracts, 32:01-10. 